\title{
On-line Energy Management for HEV based on Particle Swarm Optimization
}

\author{
S. Caux*, D. Wanderley-Honda*, D. Hissel**, M. Fadel* \\ * LAboratoire PLAsma et Conversion d'Energie \\ LAPLACE UMR 5213 CNRS ; INPT ; UPS - 2 rue Camichel - 31071 Toulouse - France \\ ** FEMTO-St - FCLAB - University of Franche-Comte UMR 6174 - 90010 Belfort - France
}

\begin{abstract}
This study considers a Hybrid Electrical Vehicle supplied by a Fuel Cell stack and supercapacitors used as Storage Element. In such an application, real time energy management is of paramount importance in order to increase autonomy and be able to deal on-line with perturbed power demand. Many offline power flow optimization principles are available but online algorithms are preferred and should be derived for optimal management of the instantaneous power splitting between the different available power sources. Based on particle swarm optimization algorithm, this study defines the parameters tuning of such algorithm. The final power splitting allows not only recovering energy braking but also is robust to some disturbances occurring during the trip. The solution provides good-quality and high-robustness results in a certain class of mission profile and power disturbance.
\end{abstract}

Key Words : Particle Swarm Optimization, Hybrid Electric Vehicle, Multi-source System.

\section{INTRODUCTION}

Replacing oil-based engines, and dealing with renewable sources is a challenge because the power distribution structure change drastically. Each decentralized production has its own properties and capacities. In transport applications when zero emission is foreseen, Hybrid Electrical Vehicles are composed with a main sources of Energy (or Power) and storage elements to be able to store and to restitute an additional energy (or power) when requested [1]. In transport applications, it is obvious that energy braking recovery should be performed and the stored energy should be provided to the powertrain not only to respond to some high power requests but also to manage the global efficiency of the electrical system. 
Maintaining the global efficiency at a maximum level with a dedicated energy management strategy allows autonomy to increase, minimizing the consumption on a given mission profile, but allows also increasing the durability of the different electrical and/or electrochemical components (Fuel Cell, Supercaps or Batteries...). As for stationary applications, a transportation application can be considered with power demand: 1-known, 2known with perturbations or 3-unknown. Considering nothing is known in advance about the power that has to be delivered in the future, it's obvious that no energy optimization can be performed; a certain level of knowledge of the power demand is also requested. With this consideration two categories of optimization principles can be listed [2]:

- off line global optimization

- on line 'partial' optimization

In fact when the whole profile to be followed is known and when the different elements are already characterized, it is possible to compute a criterion to be minimized off-line by existing algorithms that work well. There is no need of dynamic consideration at this decision level, the computation search an optimal splitting not so quickly with regards to electric or fluid mechanics time constants. All controlled elements (Fuel Cell Current/Pressure/Flow and Supercaps Current/Voltage) are considered able to follow this (details can be found in [15]).

In this category, Dynamic Programming (D.P.) is one of the most used algorithm. Its principle is based on the Bellman's principle [3] and starts computation from the end of the profile to find an optimal path reaching the beginning and leaving the optimization criterion as low as possible. Then, the founded path must be replayed. Some considerations should be given to having an accurate solution but problems arise when constraints are added and when computer time computation is limited.

Optimal control is also use to replay a sequence of control computed optimally off-line using Pontryagin principle [4]. This technique provides good results when the criterion can be expressed linearly and thus derived. Constraints are also not obvious to include and some parameters are hard to be tuned to obtain the solution. 
In both cases when the power request changes, the sequence to be replayed is not the one's that has been considered for the optimization process and implies, in most cases, an increase in the forecast fuel consumption.

Real time energy management strategies are mainly based on linguistic rules or artificial intelligence [5],[6]. Commonly used in HEV, logical rules are quite easy to define, when considering the management of 2 or 3 sources. These rules impact directly on the consumption and must be optimized [7], [8]. Fuzzy-Logic supervisor, Neural Networks [6], System with Multi-Agent [5] or adding Genetic Algorithm [16] are also used. All methods suffer from the same problems: complexity is increasing and all methods are based on learning patterns.

In this paper a stochastic on-line optimization principle is used. Particle Swarm is a method to explore a given space where an optimal solution is sought. In this case several iterations will ensure that the optimal solution is found. But the computing time can be limited (when the number of iteration is fixed, or when the number of considered particles is low for example). Therefore, a set of parameters can be tuned to reach the solution while keeping a low computation time. When the power splitting should be refreshed with a certain periodicity, this kind of algorithm can provide a sub-optimal solution or even reach the optimal one.

In part II, the HEV powertrain is described and all characteristics of the different onboard sources are given. In part III, the problem formulation is detailed to minimize the criterion and to respect all listed constraints. In part IV attention is focused on advantages of the proposed particle swarm algorithm. Part V presents the complete study of parameters tuning and results obtained using different actual profiles, with or without perturbations. Analysis, limitation and conclusion are given in Part VI.

\section{PROBLEM DESCRIPTION}

\section{A. Power flows and Energy Onboard}

In this paper a classical Hybrid Electrical Vehicle is considered. Different power trains can be defined and sizing of all elements should be optimized as well [9],[10]. To not treat 'systemic' problem, a given hybridization is fixed here and only two electrical sources are linked to the same DC bus with their own choppers. Fig1 describes the considered serial electrical structure. It can be noticed that an electrical node 
appears between the different subsystems.

The Hydrogen Tank, the Fuel Cell and its ancillaries (Compressor, Valves and local control...) and the non reversible chopper are grouped constituting the Fuel Cell System and considered as the primary sources FCS.

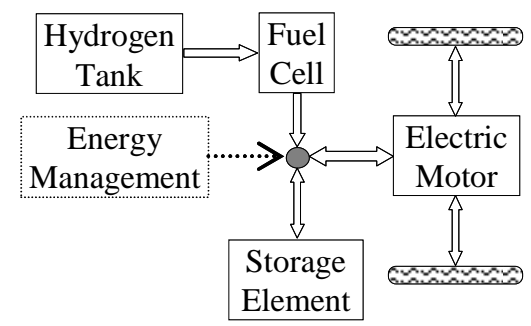

Fig 1: serial electrical structure for HEV with FC and SE

On the other hand, Supercaps Elements and the reversible buck/boost chopper allow constituting a second electrical source - SSE - which can store or provide energy.

The moto-propulsion group is here simplified because only the electrical power demand is considered. With this consideration if the powertrain architecture changes (boggy or wheels, synchronous or DC machine...) only the electrical power demand should be computed when leaving all the other elements in the optimization algorithm.

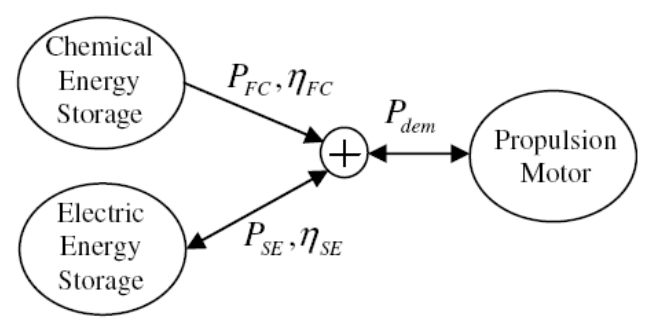

Fig 2 : Multi-source system problem formulation

Fig 2 presents the composition of the 2 main sources focusing on different efficiencies (losses) which should also be easily adapted if some element is changing (different FC size, different switching components in chopper etc.). Fig 2 also represents the electrical node and the power and efficiency data requested to be able to run an optimization algorithm in such an application.

\section{B. Efficiency of the main elements}

Algorithms, solving the optimal energy management problem are fed with data describing the efficiency of all elements. Each source has its own behavior meaning that it presents a different efficiency depending on 
the power delivered [11],[12]. Local controls are considered effective. Fuel Cell Stack stay at a given temperature and pressure and hygrometry are supposed to be well-maintained [13],[14]. Homogenous current repartition in the FCS allows dealing with a global FCS efficiency behavior $\eta_{F C}$, described in Fig 3. Storage element is commonly made with several commercial supercaps in serial and parallel to obtain the System Storage Element (SSE). Balance between supercaps elements is also considered effective and just one constant global equivalent resistance $R s c$ and capacitance $C s c$ are considered with losses only depending on losses in chopper, therefore SSE global efficiency $\eta_{S E}$ is given in Fig 4.

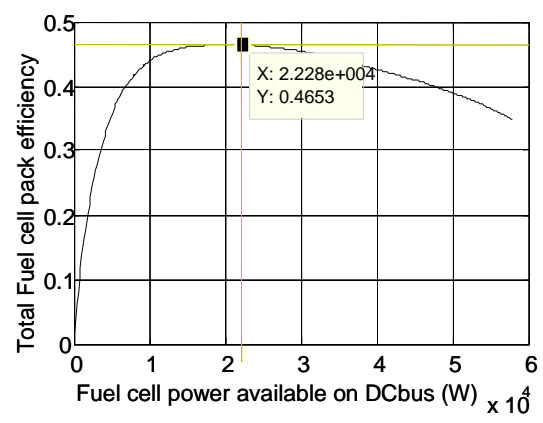

Fig 3: Fuel Cell system global efficiency curve

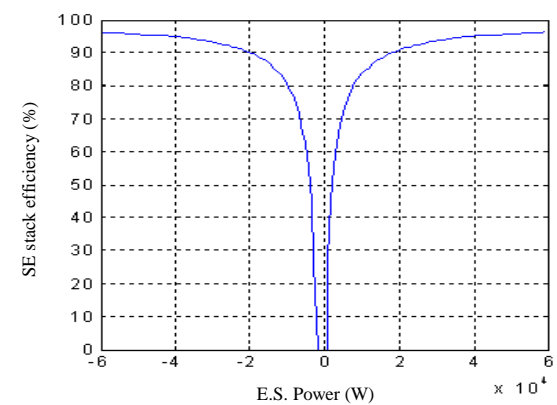

Fig 4 : Reversible Storage Element global efficiency curve

Note that the Maximum Efficiency Point for a Fuel Cell System is allocated at about $25 \%$ to $30 \%$ of the nominal power $(22 \mathrm{~kW}$ here for a $60 \mathrm{~kW}$ FCS).

Fig 3 and 4 provide the efficiency maps to be considered by the algorithm to compute the efficiency of the powertrain through a criterion to be minimized.

Moreover some constraints should also be verified because FCS and SSE have a given power size limitation and also a given Min and Max State Of Charge to avoid limited durability or even destruction. A condition fixing equal initial and final State Of Charge is commonly added in order to compare the control strategies each other. 
The optimal use of the energy braking recovery is a good way to minimize the vehicle consumption. Using the considered power profile, an a priori sizing as been made and is given in Table I. The two power demands used are presented in fig 5 - INRETS is an urban profile for a personal vehicle and fig 6 ESKISEHIR is the name of a power profile measured on a tramway line in Turkey [14],[15].

TABLE I

\section{SYSTEM POWER AND ENERGY CONSTRAINTS}

\begin{tabular}{|c|c|}
\hline \hline Symbol & $\begin{array}{c}\text { Quantity } \\
\text { Value }\end{array}$ \\
\hline \hline PSEmin & $-60 \mathrm{~kW}$ \\
\cline { 1 - 1 } PSEmax & $60 \mathrm{~kW}$ \\
\cline { 1 - 1 } PFCmin & $0 \mathrm{~kW}$ \\
\cline { 1 - 1 } PFCmax & $70 \mathrm{~kW}$ \\
\cline { 1 - 1 } SOEmin & $400 \mathrm{~kW} . \mathrm{s}$ \\
\cline { 1 - 1 } SOEmax & $1600 \mathrm{~kW} . \mathrm{s}$ \\
\hline
\end{tabular}

This sizing is made to let the power splitting explore all possible solutions and let emerge if possible solutions using only FCS or only SSE, or all combination to supply the power demand. No 'systemic' hybridization linked to size or weight is considered in this approach [10],[12] to provide efficient algorithms whatever the HEV characteristic is.

Considering a given requested power profile (cf. figures 5 and 6), this paper proposes to respond to the following question: what is the optimal power splitting in order to obtain a maximal efficiency of the vehicle (and thus the lowest fuel consumption) on the driving cycle?

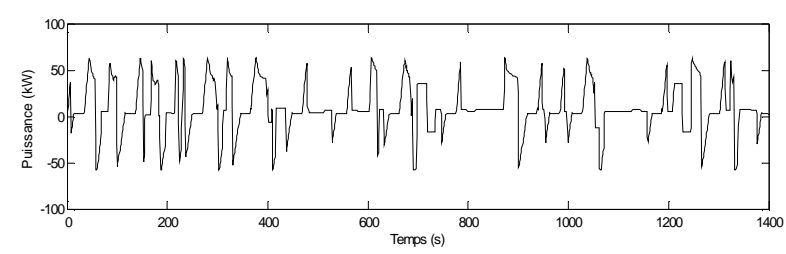

Fig. 5. INRETS : Power profile of a hybrid vehicle in urban area

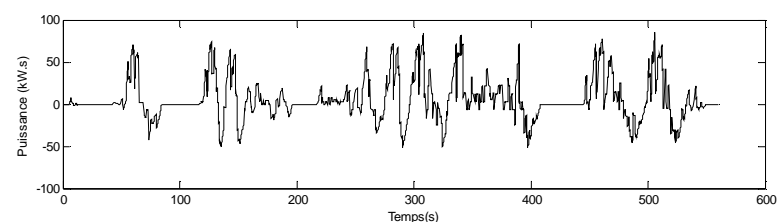

Fig. 6. ESKISEHIR : Power profile of a tram on the line of Eskisehir (Turkey) 
Using the complete knowledge about these profiles allows running off-line optimal algorithm (based for example on Dynamic Programming DP). Consumption given in table II shows that more than $30 \%$ of consumption can be earned on a given profile using optimally the storage element, instead off no storage capability if Fuel Cell is alone. These results are only provided here as reference and dynamic programming is well known [3] but only computable off-line and not in real time.

\section{TABLE II}

Energy storage improvement

\begin{tabular}{|c|c|c|c|}
\hline & D.P. & F.C. alone & Gain \\
\hline INRETS & $9189.7 \mathrm{~kW} . \mathrm{s}$ & $14891 \mathrm{~kW} . \mathrm{s}$ & $38.28 \%$ \\
\hline ESKISEHIR & $31826 \mathrm{~kW} . \mathrm{s}$ & $48043 \mathrm{~kW} . \mathrm{s}$ & $33.75 \%$ \\
\hline
\end{tabular}

\section{ALGORITHMS AND ENERGY MANAGEMENT}

The energy management problem is formulated here as a global dynamic optimization problem under constraints. The hydrogen consumption is quantified as a cost function to be minimized. The cost function is evaluated over a defined period of time.

The system's dynamic equation is:

$\dot{E}(t)=-P_{S}(t)$

Where the energy level stored $E$ is the state variable and the power $P_{s}$ the control variable.

The cost function to minimize is the "total consumed energy" of hydrogen $E_{\mathrm{H}_{2}}$ over a period of time $\left[t_{f}-t_{i}\right]$.

$E_{H_{2}}=\int_{t_{i}}^{t_{t}} \frac{P_{F C}(t)}{\eta_{F C}\left(P_{F C}(t)\right)} d t$

Energy $E_{\mathrm{H}_{2}}$ consumed is the integration of power $P_{F C}$ in the fuel cell side (not in the DCbus side where there is the power demand $P s)$ so the efficiency of the Fuel Cell Stack $\eta_{F C}\left(P_{F C}(t)\right)$ (inverter and also the fuel cell behavior provided by fig3) is introduced to 'modelized' the losses of energy passing through the FCS.

Using the previously defined efficiency, the cost criterion is therefore:

$$
\gamma=\frac{P_{F C}(t)}{\eta_{F C}\left(P_{F C}(t)\right)}
$$


The system is subject to non linear constraints of inequality related to the constraints linked to the design of the stack, the storage element power and state of charge resumed in (3):

$$
\begin{aligned}
& P_{S E_{-} \min } \leq P_{S E_{1}}(t) \leq P_{S E_{-} \text {max }} ; P_{F C_{-} \min } \leq P_{F C}(t) \leq P_{F C_{-} \max } \\
& E_{\text {min }} \leq E(t) \leq E_{\text {max }}
\end{aligned}
$$

Satisfying the power demand imposes an equality constraint (4):

$P_{S E}+P_{F C}-P_{d e m}=0$

An additional condition (5) is imposed artificially in order to ensure that the state of charge is maintained at the end of the cycle and to facilitate the optimization procedure and cycling the power demand without any more consideration from an energy management point of view.

$$
E\left(t_{f}\right)=E\left(t_{i}\right)
$$

\section{A. Off line principles : Dynamic Programming (D.P.) and Optimal Control (O.C. )}

The principle of Dynamic Programming (D.P.) is based on the Bellman's principle [3]. This principle can be resumed saying: if an optimal path reaches a path which is optimal the total path is optimal. So the algorithm starts computation from the end of the profile to find an optimal path reaching the beginning and letting the considered optimization criterion as low as possible during the optimal path.

Therefore the power demand is sampled both in time domain and in energy level. The algorithm computes at each time $t_{k}$ all possible solution linking level $E_{k-l}$ to optimal level $E_{k}$ minimizing the criterion. All results and paths must be stored and the optimal path is selected only when the algorithm reaches the origin $k=0$ with the fixed initial condition $E(0)$. So, it is clear that accuracy depends on the sampling and when the profile is long or when the energy level is divided in little steps, the computation time is exploding. Problems arise critically when non linear constraints are added and when computer time computation must be limited to respect to real time power reference generation.

Off-line optimization provides the optimal power splitting which provides the minimal criterion if replayed with the same power to follow. So the whole profile must be known in advance and unchanged to stay optimal 
and this solution is commonly used to give the optimal reference only for comparison purposes with other algorithms.

It can be noticed that Optimal Control (O.C.) theory is another way to compute the optimal control off-line and to be replayed. This technique using Pontryagin principle provides good results when the criterion can be expressed linearly and thus derived [4]. This optimization based on the minimization/cancelation of the Hamiltonian of the criterion shows linear and derivation assumption problems. Constraints are difficult to include, and some parameters dealing with Lagrange Multiplier are hard to be tuned to obtain the solution.

In both cases, when the power demand changes, the sequence to be replayed is not the correct one and let the fuel consumption increase and sometimes the offline sequence provides a worse result than even the control proposed considering no optimization at all. All the study should be done again even if the disturbance has little magnitude or is only local.

\section{B. Online principles : Rules-Fuzzy-AI}

Based on expert knowledge, logical rules are easy to establish. In fact it is easy to say the system should use the FC when SE is empty or should use both FC and SE when power is high and SE in its average State Of Charge etc.

These rules are impacting directly on the consumption and the expert should come up with a way to pass from one rule to the other. So the fuzzy approach is a solution to define each rule, the membership functions and the universe of discourse [7]. Moreover position of all membership functions can be optimized on a given profile. In fact, Genetic Algorithm [15] or Neural Network [6] can 'learn' the profile and the optimization is still made off-line on a given profile but the supervisor build in this way is able to propose an optimal or near-optimal solution even if the profile is not exactly the one known.

Artificial intelligence and expert analyzes should be mixed to have not only an accurate optimal solution, respecting constraints but also an algorithm keeping under control the complexity and the computer time requested to reach the solution in real time.

\section{On-line stochastic : Particle swarm}

Considering that computing all solutions is not possible online and in real time (with actual processor). Considering that rule-based algorithm must still be optimized offline and using them online can sometimes 
not respect important constraints, the idea is here to use both advantages using particle swarm optimization principle. Particle Swarm Optimization (PSO) principle is based on (6) and (7):

$$
\begin{gathered}
\vec{v}_{k+1}=\vec{a} \otimes \vec{v}_{k}+\vec{b}_{1} \otimes \vec{r}_{1} \otimes\left(\vec{p}_{1}-\vec{x}_{k}\right) \\
+\vec{b}_{2} \otimes \vec{r}_{2} \otimes\left(\vec{p}_{2}-\vec{x}_{k}\right) \\
\vec{x}_{k+1}=\vec{c} \otimes \vec{x}_{k}+\vec{d} \otimes \vec{v}_{k+1}
\end{gathered}
$$

Where $\vec{v}$ is a vector of particles speed, $\vec{x}$ their positions and $\otimes$ represents terms by terms vector multiplication [16],[17].

This stochastic algorithm explores randomly the space of solution and depending on the number of particles and the number of iterations made, engineer can fix accuracy and limit the requested computation time.

This evolution (6) is based on bee behavior (random flights of bee swarm) and to the optimal solution after some iteration $k$-> infinity, the swarm conserves the better value seen in the previous iteration $p l$ and the best value ever seen $p 2$ to update velocities and thus particle positions. Attracting coefficients $a, b, c, d$ ensure exploration and convergence form (zigzagging, oscillating, exponentially converging...). In this considered classical case (no abrupt optimal or sub-optimal minima) values found in literature are used: $a=0.729, b=1.494$ and without loss of generally $c=1, d=1$ and $r 1$ and $r 2$ are randomly chosen in [0 1], $[16],[17]$.

\section{PARTICLE SWARM AND ON-LINE HEV ENERGY MANAGEMENT}

In the HEV online energy management problem, the number of particles $n b \_p a r t$, the number of iteration $n b \_i t$, and the number of swarm $n b \_s w a r m$ should be fixed. Moreover to limit the number of calculus and the computing time, the problem should be reduced to an optimization in a given window size, so, the whole power profile demand should be divided. Fig7 shows the different loops requested by P.S.O. algorithm (defined with nb_swarm, nb_it values), and also presents the parameters that define the complexity of the problem to be solved (window'size, nb_part). The values of each particle change iteratively to found the optimal path in the windows defined by $\left[E k E_{k+1}\right]$. 
A certain number of particle $n b \_p a r t$, is fixed randomly at different energy level at each time in a window $[t k t k+1]$. The sampling time $\Delta t$ and window's size increase the computation time. All particles are free to explore all energy level from [0 100\%] with a step of about $1 \%$, this $\Delta E$ is chosen to be as accurate as possible taking into account the possible chopper and source capacity to maintain $E_{k}$ and also to preserve a realistic computation time.

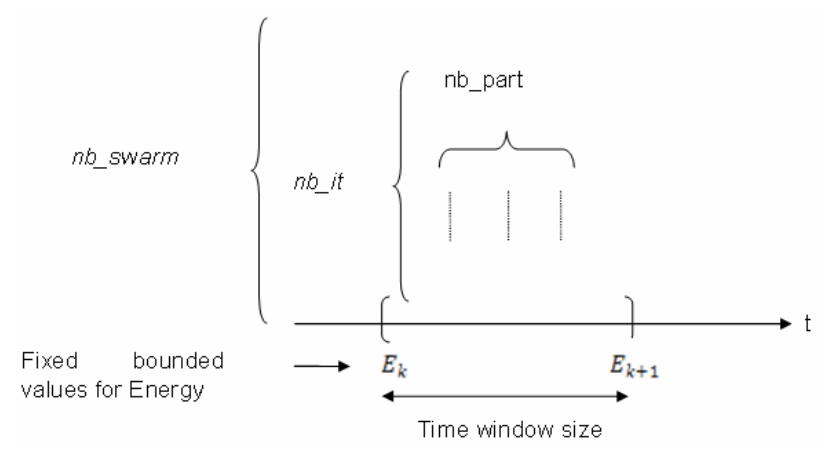

Fig 7 : set of parameters of the particle swarm algorithm

The criterion is minimized on the window size and consumption is minimized optimally using energy levels $E_{k}$ and $E_{k+1}$ equal to the one computed by Dynamic Programming algorithm. It can be noticed that this approach mixes the offline optimization and the online minimization which can be seen as an adaptation capacity. In fact, if no disturbance occurs, particle swarm optimization should converge to the same path as Dynamic Programming. In case of disturbance, the rejection is ensured by particle swarm to locally change the path and return, if possible, to the optimal energy level $\mathrm{E}_{k+1}$ that ensure an optimal ending (with no more disturbance).

All set of parameters can be tested on a profile in a first step to be roughly defined. The correct set should ensure almost $50 \%$ of swarm funding the optimal path and delivering a result in a time lower than the refreshing reference period $\mathrm{t}_{k+1}-\mathrm{t}_{k}$.

\section{A. Simulation based tuning}

Running some simulations with different sets of parameters allows establishing the values shown in Table III. Clearly, the higher are the $n b \_s w a r m$ or $n b \_i t$ or $n b \_p a r t$ higher is the computation time but the higher is the probability to find the best path. If 3 on 5 swarms have found the optimal path means the path is found with $60 \%$ of chance, therefore the path can be considered always found. It can also be seen that using 5 
swarms and 20 particles is correct because this set provides the optimal path with $60 \%$ of chance in a low and feasible actual computing time. Lower values provide less than $50 \%$ path found and are considered too risky to be used in the real time optimization problem.

The code should be also optimized so values given in Table III should be used only in a relative way.

NB: program executed in Matlab® R2007a on a Windows XPpro environment dedicated to calculus (processor: PowerEdge 6850, Quadri Xeon, 3.2GHz, 32Go Ram, 2x150 Go Scsi UTRA 320).

\section{TABLE III}

Set of parameters and solution found

\begin{tabular}{|c|c|c|c|c|}
\hline$n b \_s w a r m$ & $n b \_p a r t$ & $n b \_i t$ & $\begin{array}{c}\text { Average } \\
\text { computation } \\
\text { time (s) }\end{array}$ & $\begin{array}{c}\text { Optimal } \\
\text { path } \\
\text { found }\end{array}$ \\
\hline 5 & 50 & 100 & $4.28 \mathrm{~s}$ & 5 \\
\hline 5 & 50 & 50 & $1.78 \mathrm{~s}$ & 5 \\
\hline 5 & 40 & 100 & $3.42 \mathrm{~s}$ & 5 \\
\hline 5 & 40 & 50 & $1.38 \mathrm{~s}$ & 4 \\
\hline 5 & 30 & 100 & $2.46 \mathrm{~s}$ & 4 \\
\hline 5 & 30 & 50 & $1.06 \mathrm{~s}$ & 4 \\
\hline 5 & 20 & 100 & $1.56 \mathrm{~s}$ & 3 \\
\hline 5 & 20 & 50 & $0.76 \mathrm{~s}$ & 3 \\
\hline
\end{tabular}

\section{B. Validation on actual profiles}

Using sampling time of $\Delta t=2 \mathrm{~s}, \Delta E=1 \mathrm{kWs}$, the power demand is satisfied and state of charge of the storage element is shown in Fig 8.

In this figure with no perturbation on the profile, it can be noticed that D.P. path is the same as the one found in each Particular swarm window, the curves obtained with fuzzy logic is only provided to see the possible different energy managements and details can be seen in [4], the use of the proposed P.S.O. on a different profile is analyzed in the next section.

It has to be noticed that both optimization provides quite the same consumption of energy on the whole profile $($ Part. Swarm/D.P.= 10362kW.s and Fuzzy= 10358kW.s $)$ but fuzzy leaves some power demands not 
furnished (due to saturation at low SOC) and is only shown for comparison purposes. Storage element is considered to be charged at $900 \mathrm{kWs}$ and respect its min and max S.O.C. during the trip and allows managing optimally the energy on board.

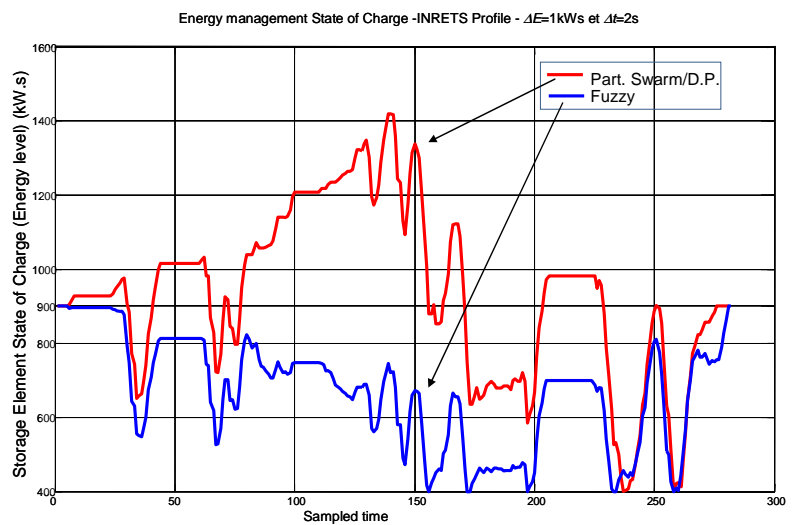

Fig 8 : Energy management State of Charge -INRETS Profile - $\Delta E=1 \mathrm{kWs}$ et $\Delta t=2 \mathrm{~s}$

\section{VALIDATION AND COMPARISON}

Previous sections demonstrate that particular swarm algorithm is capable of finding the optimal, windows by windows, using the offline energy level computed for example with D.P on a given profile as a reference. This section tests the proposed algorithm on different profiles and with an unknown perturbation added artificially to characterize the robustness of such an approach.

\section{A. First comparison}

In fact, depending on the power profile, parameter adjustment must be made. Of course, $n b \_p a r t$ and $n b \_i t$ are linked to the convergence velocity and thus the possibility to adapt the particle speed quickly to the profile variation. So using ESKISEHIR profile instead of INRETS with the set of parameter $n b \_p a r t=50$, $n b \_i t=50, n b \_s w a r m=5$ is not sufficient. By analyzing where the problems are located, it was noticed that no solution where found in some specific windows (the other are computed without any problem). For these windows the power variation is greater as in INRETS profile and is shown in Fig9. This profile presents some section with a higher power variation so the $n b_{-}$part or $n b_{-} i t$ must be increased to be able to find the optimal path. So as for many other methods, analyzing the power demand in terms of max power, mean power and power variation demand, not only provides sizing information but also information to tune accurately optimization parameters in the algorithm. 

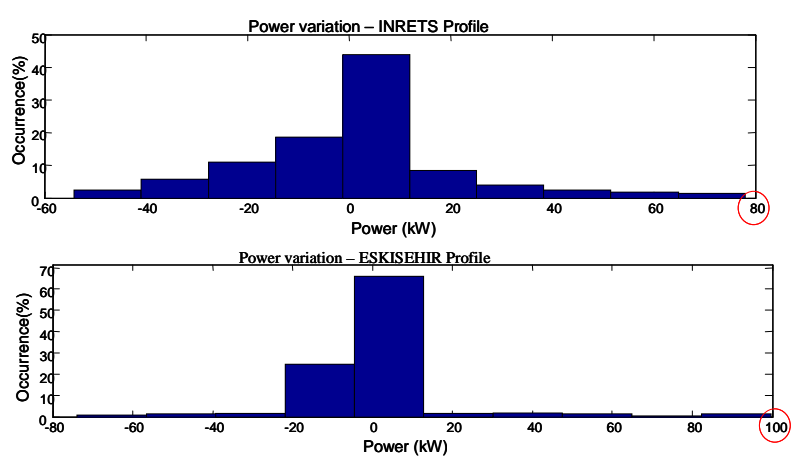

Fig 9 : Power variation occurrence in different ranges of magnitude

Using $n b \_p a r t=20, n b \_i t=50$, nb_swarm $=7$ is convenient for both profiles. The same study should be made on other profiles to be sure to be able to optimize the criterion.

Even if the optimization is not optimal, the particle swarm algorithm provides a solution better than optimization following offline references. Adding a perturbation on a given profile may justify the adaptation ability of particle swarm algorithm.

\section{B. Performance and robustness comparison}

To verify the possible adaptation of the proposed algorithm, a perturbation is added to the known power demand INRETS limited to the $50^{\text {th }}$ first point (50s) Fig11. In fig 11 a non expected positive power demand is added during 5s (higher acceleration required for example due to the road traffic). In fig 11 bis the disturbance is proposed to be negative to simulate an unexpected braking. The perturbation occurs during $5 \mathrm{~s}$ which is the size of the optimization window; tests could be made for longer disturbances but are out of scope of this paper.

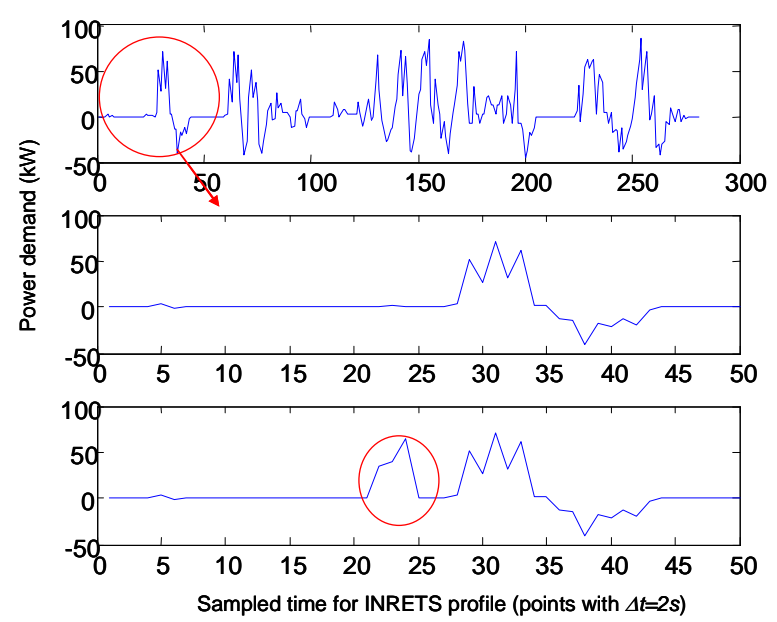

Fig 11 : Initial profile, sequence selected and first disturbance injected Dist=50kW (case dist $>0$ ). 


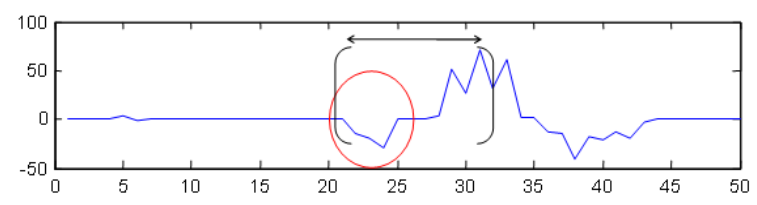

Fig $11 \mathrm{~b}:$ second disturbance type $:$ Dist $=-40 \mathrm{~kW}($ Case dist $<0)$

From the start point 0 to point 50 , with no disturbance, the optimal energy consumption is $790 \mathrm{kWs}$, with positive disturbance used, the consumption is $1404 \mathrm{kWs}$ and when the unexpected braking energy is used (negative disturbance) the consumption is $592 \mathrm{kWs}$.

Depending on the position and here mainly on the perturbation magnitude, it is possible or not to optimize the criterion in a given window size. In the case under study, the profile does not present high power demands after the disturbance point [25-28] and the energy recovered can not be delivered in the foreseen windows. Moreover, if the energy stored is quickly used in this phase, the Fuel Cell power may decrease, decreasing its efficiency, thus a bad criterion is obtained. Using a window twice larger (Fig 11b), the algorithm can find a solution to return to the energy state imposed respecting constraints of the system. Increasing the optimization window size means increasing the computation time and the necessity to verify real time constraints. $n b \_s w a r m=1$ solves partially this issue but 4 tests on 10 provides no optimal path (only with an increase of fuel consumption limited to $1 \%$ ).

TABLE IV

Set of parameters and robustness

\begin{tabular}{|c|c|c|c|}
\hline & Ideal & Dist $>0$ & Dist $<0$ \\
\hline $\begin{array}{c}\text { Window size } \\
\text { (nb points) }\end{array}$ & 5 & 5 & 12 \\
\hline nb_swarm & 7 & 1 & 7 \\
\hline nb_part & 20 & 20 & 100 \\
\hline nb_it & 50 & 50 & 50 \\
\hline
\end{tabular}


This last study means that an adaptation is requested on line when a disturbance is detected to be able to switch the strategy (different set of parameters for the particular swarm - resumed in Table IV) to obtain a good result. Of course, a prediction or a statistical data-base can be used to classify the different possible disturbances as well as the requested analysis to classify the profile.

\section{CONCLUSIONS}

Particle swarm optimization is an efficient solution for on-line energy management for Hybrid Electrical Vehicle. It did not pass over common problems in such application: the profile must be studied and classified to find accurate set of tuning parameters. Performances are better when references are provided with off-line global optimization. Disturbances must be predicted or a degree of freedom must be used to cancel their influences.

This approach is not really an Artificial Intelligence approach using learning phase, but using optimal references, the particular swarm optimization algorithm is able to compute, in a limited computation time, the optimal path in terms of fuel consumption.

Particles are able to compute in real time the optimal path with some capacity to reject disturbances and in each case to adapt the optimal path to a sub-optimal path respecting real time computing constraints. Implementation on an actual vehicle should be made in a next step in order to validate the approach.

\section{AKNOWLEGMENT}

INRETS: to provide actual measurements obtained in actual vehicle and trip, and interests given in energy management in transport application.

FEMTO-ST / FCLAB: to provide actual data obtained on fuel cell test bench developed in Belfort (France) and collaboration in this study.

\section{REFERENCES}

[1]L. Solero, A. Di Napoli, and al. : "Fuel cell HEV's assisted by ultracapacitor and battery storage system", FISITAHelsinki, Finland - June 2-7 2001.

[2]F. R. Salmasi, "Control strategies for hybrid electric vehicles: Evolution, classification, comparison, and future trends," IEEE Trans. on Vehicular Technology, vol. 56, no. 5, pp. 2393-2404, Sept. 2007.

[3] R.E. Bellman : 'Dynamic Programming'. Princeton, NJ, USA-1957, Princeton University Press. 
[4] S. Delprat, J. lauber, T. Guerra, and J. Rimaux, "Control of a parallel hybrid powertrain: Optimal control," IEEE Trans. on Vehicular Technology, vol. 53, no. 3, pp. 872-881, 2004.

[5] R. Langari, and J.S. Won, "Intelligent energy management agent for a parallel hybrid vehicle - part I: System architecture and design of the driving situation identification process," IEEE Trans. on Vehicular Technology, vol. 54 , no. 3, 2005.

[6] J. Moreno, M. E. Ortúzar and J. W. Dixon : 'Energy-Management System for a Hybrid Electric Vehicle, Using Ultracapacitors and Neural Networks' - IEEE Trans on Industrial Electronics, vol. 53, N. 2, april 2006 pp614623.

[7] D. Gao, Z. Jin, Q. Lu : 'Energy management strategy based on fuzzy logic for a fuel cell hybrid bus', Jour. Power Sources, Volume 185, Issue 1, (15 October 2008), Pp 311-317.

[8] M. Tekin, D. Hissel, M.C. Péra, J.M. Kauffmann, “Energy management strategy for embedded fuel cell system using fuzzy logic”, IEEE Transactions on Industrial Electronics, vol. 54, n¹, pp. 595-603, 2007

[9] M. Hatti, M. Tioursi : 'Dynamic neural network controller model of PEM fuel cell system', Int. Jour. of Hydrogen Energy, Vol 34, Issue 11, June 2009 - Pp 5015-5021

[10]M. Ehsani, K.M. Rahman, H.A. Toliyat : 'Propulsion system design of electric and hybrid vehicles.'; IEEE Trans. On Industrial electronics - vol 44, Issue 1, Feb. 1997 - pp:19 - 27

[11] M. Tekin, D. Hissel, M.C. Pera and J.M. Kauffmann : "Energy optimization of a Fuel Cell Generator : Modelling and experimental results” ”, European. Power Electronic conf. EPE’03, Toulouse - France, CDRom

[12] G. Fontes, C. Turpin, R. Saisset et al. : "Interaction between fuel cells and power converters influence of currentharmonics on a fuel cell stack. “, proc of Power Electron. Specialists Conf. PESC04 - 20/25 june 2004 Aachen - Germany

[13] V. Naso, M. Lucentini and M. Aresti: "Evaluation of the overall efficiency of a low pressure proton exchange membrane fuell cell power unit” Americ. Inst. of Aeronautics and Astronautics AIAA 2000 pp1147-1150

[14] S.Caux, J.Lachaize, M.Fadel, P.Schott and L.Nicod PEMFC : "Air Loop Model and Control.” Conference Vehicle Power Propulsion, VPP’05 - 7-9 septembre 2005 - Chicago - Illinois - USA

[15] S.Caux, J.Lachaize, M.Fadel, P.Schott and L.Nicod : "Modelling and Control of a Fuel cell System and storage elements in transport Applications.” Journal of Process Control - JPC Vol 15/4 pp 481-491

[16] W. Hankache, S. Caux, M. Fadel, D. Hissel "Real Time Fuzzy Energy Management of Fuel Cell and Ultracapacitor Powertrains" Fundamentals and Developments of Fuel Cell Conference 2008 - FDFC2008, December $10-12^{\text {th }}, 2008$ - Nancy - France.

[17] M.C. Kisacikoglu, M. Uzunoglu, M.S. Alam : 'Load sharing using fuzzy logic control in a fuel cell/ultracapacitor hybrid vehicle' - Int. Jour. of Hydrogen Energy, Vol 34, Issue 3, February 2009,pp 1497-1507

[18] I.C. Trelea : 'The particle swarm optimization algorithm: convergence analysis and parameter selection.', Information Processing Letters $n^{\circ} 85$ (2003) pp317-325

[19]J. Kennedy, R.C. Rberhart : 'Particle swarm optimization', Proc. Conf. on Neural Network 1995, PiscatawayNewJersey, USA, pp1942,1948 